\title{
Specialized protrusions coordinate migratory border cell cluster cohesion via Scribble, Cdep, and Rac
}

\author{
Joseph P. Campanale ${ }^{\mathrm{a}, \mathrm{b}}$, James A. Mondo ${ }^{\mathrm{a}, \mathrm{b}}$, and Denise J. Montell ${ }^{\mathrm{a}, 1}$ \\ ${ }^{a}$ Molecular, Cellular and Developmental Biology Department, University of California Santa Barbara, Santa Barbara, CA 93106, \\ USA. \\ ${ }^{\mathrm{b}}$ These authors contributed equally \\ ${ }^{1}$ Correspondence: dmontell@ucsb.edu
}

\begin{abstract}
Summary
Apicobasal polarity is a defining characteristic of epithelial cells and its disruption is a cancer hallmark. Distinct apical and basolateral protein modules antagonize each other to establish separate membrane domains. These modules interact with dozens of potential effector proteins. Here we describe polarity protein localization and function within a migrating epithelial cell cluster and identify a functionally significant effector protein. In Drosophila egg chambers, border cells delaminate from the follicular epithelium and migrate collectively. We report that the basolateral protein Scribble is required for border cell cluster cohesion and migration. The basolateral module localizes the Rac guanine nucleotide exchange factor Cdep to membranes, and Cdep knockdown phenocopies Scribble cluster cohesion defects. Remarkably, membrane targeting of Cdep is sufficient to partially suppress multiple Scribble phenotypes. We describe specialized basolateral protrusions that promote cluster cohesion. Scribble restricts these protrusions from encroaching onto the apical domain. Thus, a major function of the basolateral module is to localize Cdep, promoting specialized protrusions, cluster cohesion, and collective migration.
\end{abstract}

Keywords: Apicobasal polarity, Collective cell migration, RhoGTPases, Scribble complex, Par complex, Rac1, FARP2, Cdep, Border cells

\section{Introduction}

Epithelial polarization is an ancient property of cells in multicellular organisms and is controlled by a conserved suite of signaling modules that interact to define apical, junctional and basolateral membranes (Bilder et al., 2003; Goldstein and Macara, 2007; Niessen et al., 2012; Thompson, 2013). The Crumbs module includes the transmembrane protein Crumbs and its intracellular binding partners Pals1 (Drosophila stardust) and PatJ (Tepass, 2012). Adherens junctions are composed of E-cadherin and its associated catenins and are positioned by the Par module proteins aPKC, Par3 (Drosophila bazooka) and Par6 (Morais-deSá et al., 2010). The Scrib module includes Scribble (Scrib), Discs large (Dlg) and Lethal giant larva (Lgl), which establish the basolateral domain by antagonizing the Par and Crumbs modules (Krahn et al., 2010). In addition to their mutual inhibition, each module scaffolds effector proteins to produce membranes with distinct functions in absorption, secretion, adhesion, or attachment to the extracellular matrix (Campanale et al., 2017; Knoblich, 2000; St Johnston and Ahringer, 2010). Establishing and maintaining apicobasal polarized epithelia is critical for tissue homeostasis (Martin-Belmonte and Mostov, 2008; Nance and Zallen, 2011; Tepass, 2012) and tumor suppression (Lee and Vasioukhin, 2008; Macara and McCaffrey, 2013; Wodarz and Näthke, 2007) in both vertebrates and invertebrates (Bilder et al., 2000; Dow et al., 2003; Halaoui and McCaffrey, 2015; Hariharan and Bilder, 2006; Santoni et al., 2020).

While the interactions amongst polarity proteins and modules have been studied extensively, their contributions to epithelial cells undergoing dynamic morphogenetic movements are less well characterized. Much attention has been given to the epithelial to mesenchymal transition in which epithelial cells dismantle apicobasal polarity and repurpose the polarity modules to generate leading/lagging polarity (Lamouille et al., 2014, Thiery et al., 2009). However, cells that migrate collectively as groups don't entirely dispense with apicobasal polarity.

Collective migrations drive normal development, wound healing, organogenesis, and tumor metastasis (Friedl and Gilmour, 2009; Friedl et al., 2012; Mishra et al., 2019a; Scarpa and Mayor, 2016; Stuelten et al., 2017). Groups can be small or large clusters, streams, or sheets. How apicobasal polarity proteins operate in the plethora of collective migrations in vivo is an open question. Precisely how polarity modules accomplish their multifarious functions is also not yet clear. Dozens of potential effector proteins have been identified (Bonello and Peifer, 2019), whose functions are mostly unknown, and different effectors may be more or less critical in stationary versus dynamic epithelia.

The Drosophila ovary has served as a model to study epithelial polarity (Bonello et al., 2021; Goode and Perrimon, 1997; Khoury and Bilder, 2020). Drosophila egg chambers consist of 15 nurse cells and one oocyte surrounded by a monolayer of epithelial follicle cells encased within a laminin-rich basement membrane (Fig. 1A). Follicle cells exhibit classic epithelial polarity with apical aPKC and lateral Scrib (Fig. 1A). During developmental stage 9, 6-10 anterior follicle cells called border cells delaminate as a cluster and migrate between the nurse cells to the oocyte (Montell et al., 1992) (Fig. 1A). Border cell clusters contain a central pair of non-migratory polar cells that are surrounded and carried by migratory cells (Montell et al., 2012) (Fig 1B-B'). 
The Crumbs and Par modules are required for border cell cluster organization and migration (Pinheiro and Montell, 2004). The Crumbs module localizes aPKC to apical cell-cell junctions. In addition, as in other collectively migrating epithelia, in the leading cell aPKC relocalizes to the leading edge to promote protrusions (Wang et al., 2018), which probe for chemoattractants and physical space and thereby steer the cluster (Mishra et al., 2019b, Dai et al., 2020). The Scrib module is also required for border cell migration, however the molecular and cellular mechanisms are unclear (Li et al., 2011; Szafranski and Goode, 2004; Zhao et al., 2008).

Here we use temporally and spatially controlled knockdown of Scrib module proteins to uncover their contributions to border cell cluster morphology and migration. We report that Scrib knockdown results in poor coordination amongst the migrating cells, ectopic protrusions, and cohesion defects that result in cluster splitting. Knockdown of a previously uncharacterized Rho GEF, Cdep, causes similar phenotypes. Rac activity is reduced in Cdep knockdown border cell clusters, indicating that Cdep is a Rac GEF. We found Cdep protein enriched on basolateral surfaces of epithelial follicle cells and migrating border cells, in a Scrib moduledependent manner. Remarkably, basolateral targeting of GFP-tagged Cdep using a basolateral anti-GFP nanobody, rescues multiple Scrib knockdown phenotypes including border cell cohesion and migration and epithelial layering. We describe dynamic basal protrusions between individual border cells and propose that Scrib, Cdep, and Rac sculpt these protrusions, which in turn promote cluster cohesion. Our results show that a major function of the Scrib module in follicle cells is to target the RacGEF Cdep to basolateral membranes.

\section{$\underline{\text { Results }}$}

\section{Border cells relocalize Scrib and Par polarity modules as they delaminate.}

Scrib module proteins relocalize in follicle cells generally and border cells specifically, during stage 9. Within the follicular epithelium, aPKC is enriched throughout apical surfaces (Fig. 1A-B”). Scrib (Fig. 1C) and Dlg (Fig. 1C') change from uniformly distributed along the lateral membrane at early

stages to enriched in the apical portion of the lateral membrane during stage 9, whereas Lgl remains uniform (Fig. 1C").

Border cells are specified at late stage 8 and begin to form protrusions as they delaminate at stage 9 (Fig. 1D-D”). During delamination,
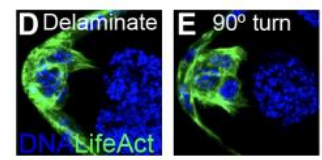
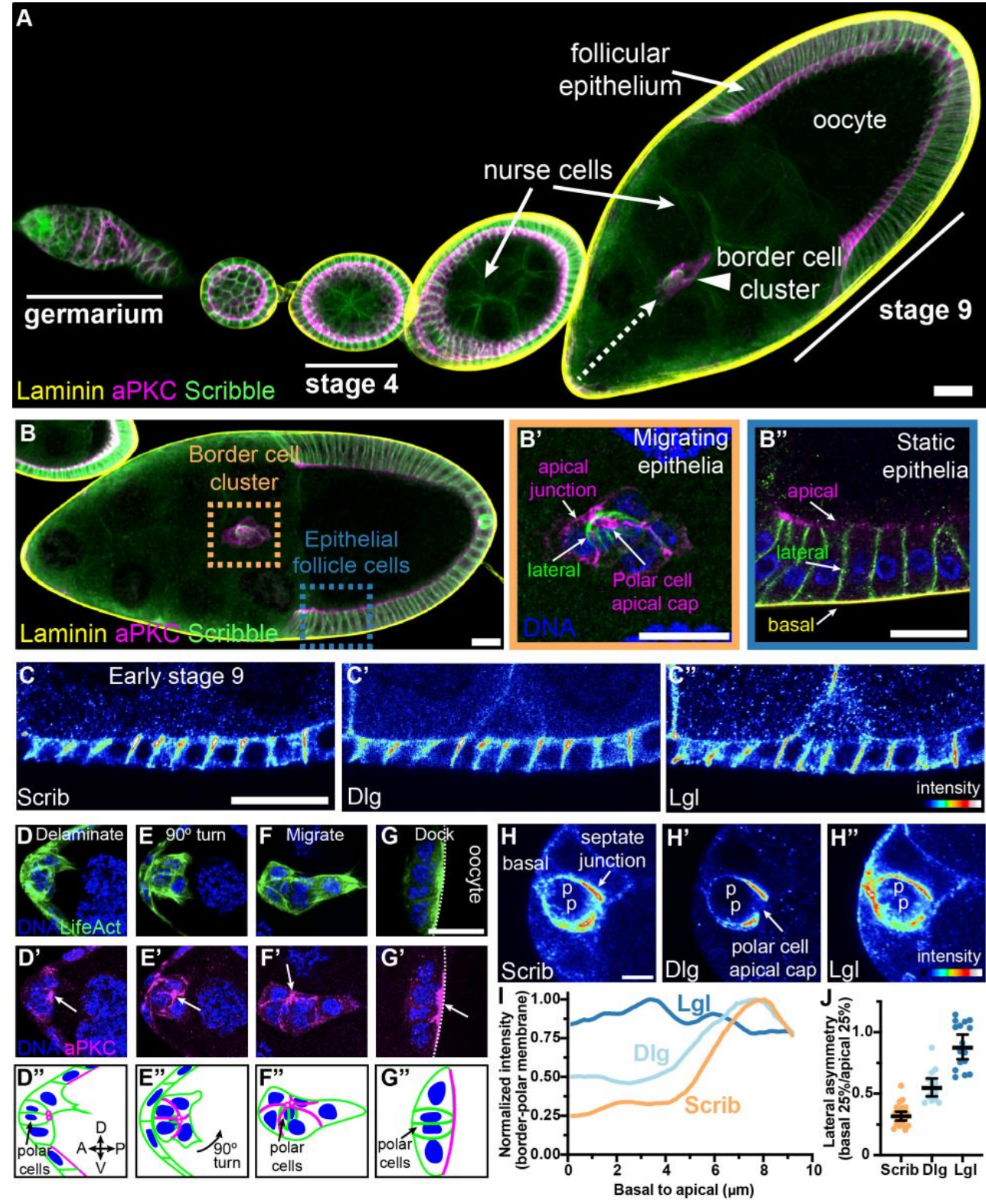

Figure 1: Border cell clusters migrate while retaining polarized Scrib module proteins. (A) Maximum intensity projected single ovariole from Drosophila ovary stained for Laminin with peanut lectin (yellow), aPKC on apical membrane (magenta), and Scribble on lateral membrane (green). (B) Projected stage 9 egg chamber with migrating border cells stained as for (A). (B') border cell and (B') epithelial follicle cell zooms of (B) with DNA (blue). (C) Epithelial follicle cells from the early stage 9 egg chamber are stained for Scrib (C), Dlg (C'), and Lgl (C"). (D-G') Stages of border cell migration from stage 9 egg chambers with DNA (blue), (D-G) Actin staining from slbo>LifeActinGFP reporter (green), (D'-G') aPKC staining (magenta) with arrows to denote apical membrane, and (D"-G") schematic representations of D-G'. Axis compass in D" indicates anterior, A, posterior, P, dorsal, D, and ventral, V. (H) Single confocal slice of a border cell cluster at late stage 8 stained for Scrib, Dlg and Lgl. Polar cell apical cap is devoid of staining ( $\left.\mathrm{H}^{\prime}\right)$. (I) Representative line scans of border cell polar/border cell membranes beginning from basal going to apical (H-H"). (J) Dot plots of basal to apical asymmetry in Scrib module staining. Each dot is a measurement from a single border cell cluster. Analysis is the basal $25 \%$ /apical $25 \%$ of the lateral membrane. All scale bars $=20 \mu \mathrm{m}$.
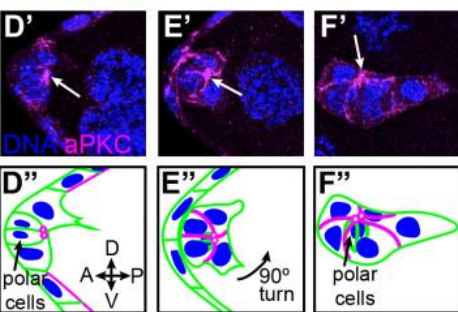

protrusions extend from the lead cell basal surface, and the cluster rotates $90^{\circ}$ such that apical membranes are perpendicular to the direction of migration and (Fig. 1E-E"). aPKC is enriched specifically at apical cellcell contacts including border cell/polar cell, and border cell/border cell 
during both delamination (Fig. 1D-E") and migration (Fig. 1F-F"). aPKC is also present at a lower level in the leading cell protrusion (Wang et al., 2018) (Fig. 1F'). Once border cells complete their migration and arrive at the oocyte, they turn $90^{\circ}$ again and dock their apical surface to the oocyte (Niewiadomska et al., 1999); (Miao et al., 2020) (Fig. 1G-G').

During delamination, Scrib (Fig. 1H) and Dlg (Fig. 1H') are enriched in the apical portion of the lateral membrane of border cell/polar cell interfaces, while Lgl (Fig. 1H") is more uniform, similar to the early stage 9 follicular epithelium (Fig. 1C', C'). Line scans from individual clusters stained for all three proteins showed that Scrib and Dlg are highly enriched sub-apically in septate junctions just below adherens junctions

while Lgl remains evenly distributed along the entire lateral membrane (Fig. 1I). Scrib module proteins are less abundant at junctions between border cells and neighboring follicle cells (Fig. 1H-H”). During migration, Scrib module proteins are also depleted from border cell/nurse cell boundaries (Fig. 1B"). In migratory clusters, the majority of Scrib and DLG is found in the apical most $25 \%$ of the lateral membrane between polar and border cells, while Lgl is more uniform along the lateral membrane (Fig. 1I). This analysis shows that border cell clusters retain distinct apical and basolateral domains, however unlike the follicular epithelium, apical proteins are restricted to cell-cell junctions and basolateral proteins are concentrated at border cell/polar cell contacts.

The Scrib module promotes cluster integrity independent of tumor suppression.

To investigate the functions of the Scrib module in border cell clusters, we expressed interfering RNA (RNAi) hairpins targeting scrib, dlg or lgl. RNAi against the white $(w)$ gene was used as a control. UASRNAi constructs were conditionally expressed in both polar and border cells using c306Gal4 (Manseau et al., 1997) together with the temperaturesensitive repressor tubGal $80^{\text {ts }}$ to provide temporal control. Scrib module proteins are tumor suppressors and mutations in these genes cause hyperproliferation and multilayering of follicle cells that at best complicate and at worst

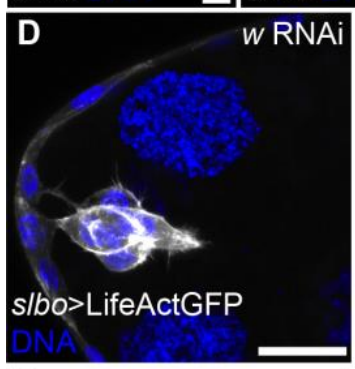

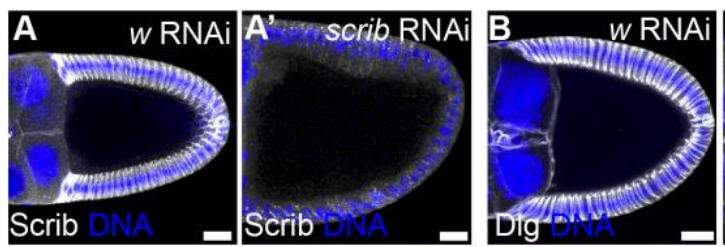
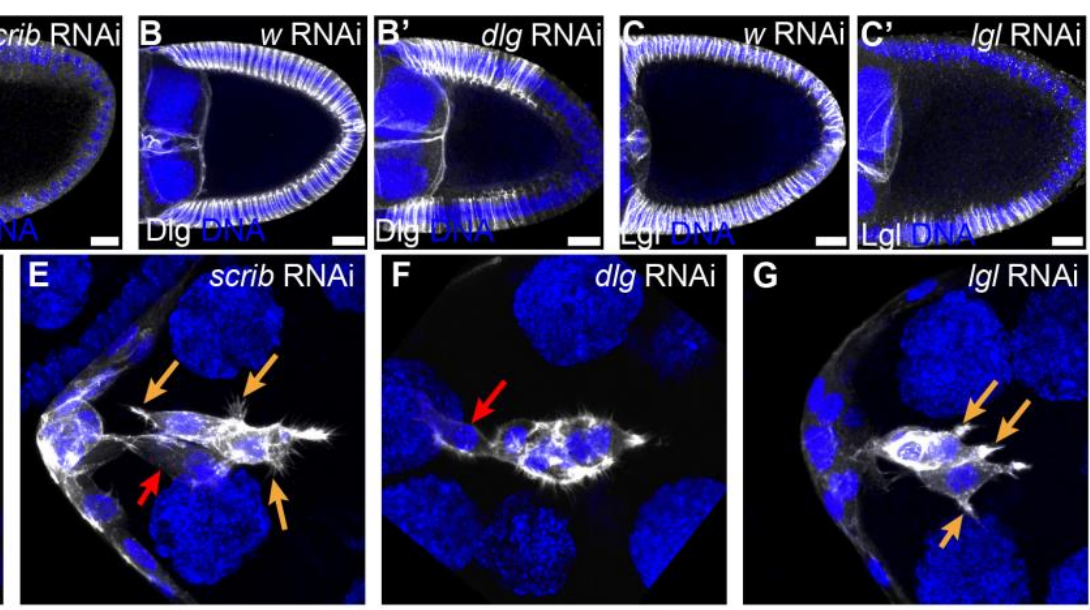

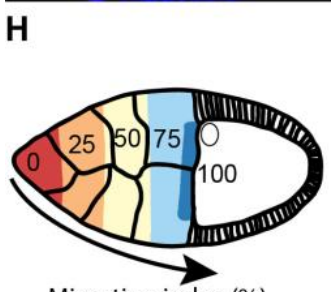

Migration index (\%)
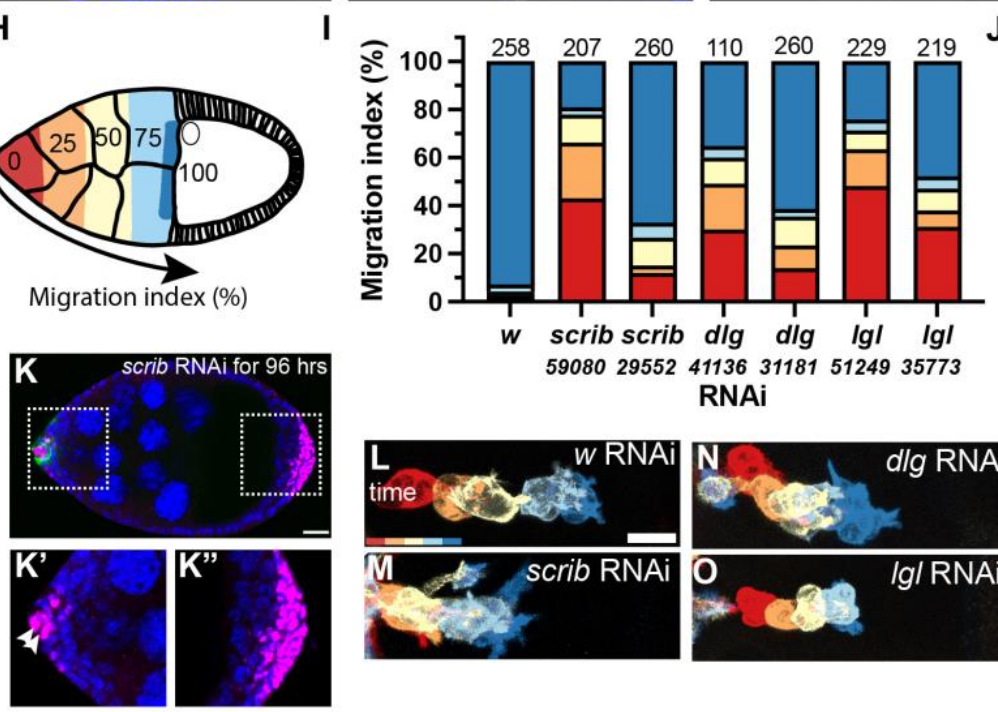

RNAi
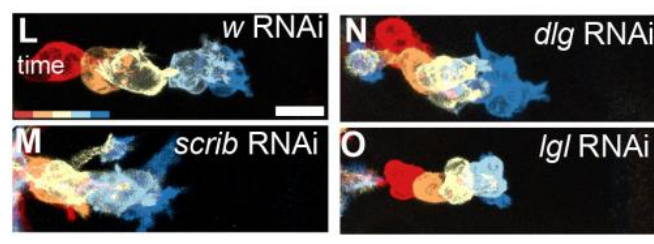
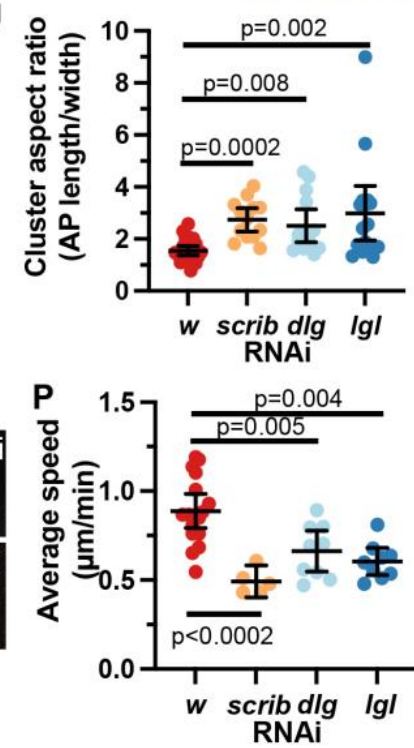

Figure 2. The Scrib module coordinates cluster cohesion, detachment, and migration dynamics. (A-C') Scrib module protein knockdowns in posterior follicle cells (gray) after expressing $w(\mathrm{~A}, \mathrm{~B}, \mathrm{C}), \operatorname{scrib}\left(\mathrm{A}^{\prime}\right), \operatorname{dlg}\left(\mathrm{B}^{\prime}\right)$, or $\operatorname{lgl}\left(\mathrm{C}^{\prime}\right)$ RNAi. (D-G) Representative maximum intensity projections of migrating border cell clusters expressing $c 306 \mathrm{Gal} 4$, slbo>LifeActGFP, tubGal80 ts, UAS-RNAi and stained for DNA (blue). Cells with ectopic protrusions noted with orange arrow and cells that fell off the cluster noted with red arrows. $(\mathrm{H})$ Schematic of migration index measurements in stage 10 egg chambers color coded. (I) Grouped column graph of migration index in stage 10 egg chambers using color color code from $(\mathrm{H})$. The number of egg chambers counted, $n$, is denoted above bars. (J) Dot plot of cluster aspect ratio. Anterior-posterior length divided by the width. Statistical significance was tested using one-way ANOVA with Kruskal-Wallace post hoc analysis. (K-K") Representative early stage 9 egg chamber expressing scrib RNAi for 96 hrs stained for cut (magenta) and DNA (blue). (P') zoom of the anterior. Polar cell nuclei marked with arrowheads. (P") zooms of the posterior. (L-O) Color coded images from $4 \mathrm{hr}$ time lapse movies for RNAi indicated. Temporal color code is a 30 min interval. (P) Dot plot of cluster migration speed. Each dot represents one movie. Statistical significance was tested using one-way ANOVA with Tukey post hoc analysis. All scale bars $=20 \mu \mathrm{m}$. 
gene caused similar effects. Longer incubation times resulted in multilayering, as expected (Fig. 2K-K"). Live imaging confirmed dynamic protrusions from follower cells and that clusters progressively elongate throughout their migration (Fig. 2L-O). Live imaging also revealed a significant reduction in the average cluster migration speed to roughly half of that observed in control clusters (Fig. 2P). These results indicate that Scrib module knockdown reduces cluster cohesion, causes ectopic protrusions in follower cells, and impedes cluster migration.

\section{Cdep is a basolateral Rac-activator that promotes cluster cohesion}

In an RNAi screen for Rho family GEFs that affect border cell morphology, we found that knockdown of Cdep caused cluster cohesion defects similar to Scrib knockdown (Fig. 3A, B). Cdep is the predicted Drosophila ortholog of human FARP2 (27\% identical and 40\% similar), a Rac GEF that promotes collective invasion of colorectal carcinoma (Libanje et al., 2019) and dendritic branching in neurons (Danelon et al., 2020; Mlechkovich et al., 2014). Live imaging revealed that compared to controls (Fig. 3A), Cdep RNAi-expressing border cell clusters splayed apart as they migrated and that follower cells fell off the cluster and/or produced ectopic protrusions (Fig. 3B) Similar to Scrib knockdown, clusters were elongated (Fig. 3C).
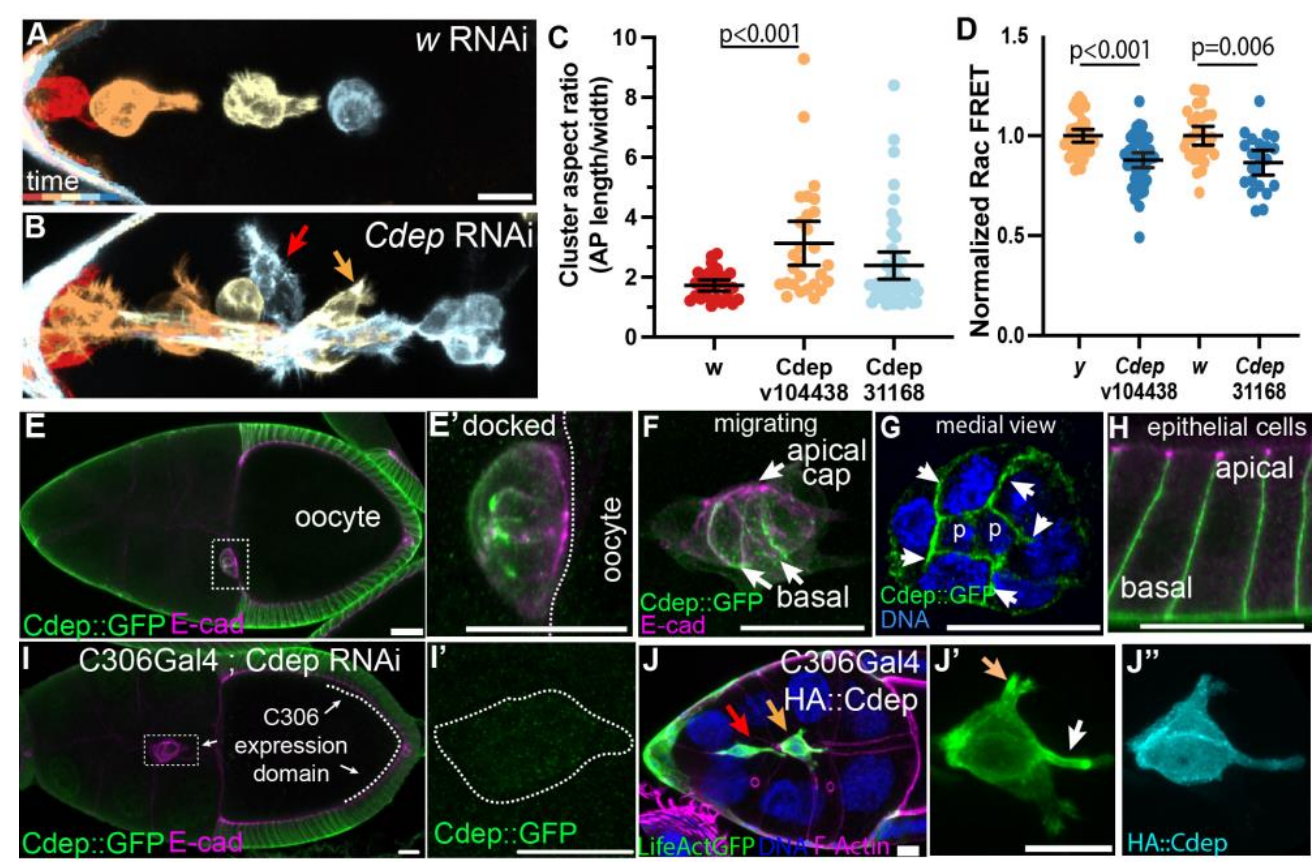

Figure 3: Knockdown of the basally localized RacGEF, Cdep, phenocopies Scrib module knockdown. (A-B) Color coded images from a 4 hour time lapse movie after $w$ or Cdep knockdown. Temporal color code set at 30 min interval. Red arrow indicates a border cell that has fallen off the cluster. Orange arrow indicates an ectopic protrusion. (C) Dot plot of cluster aspect ratio as measured by anteriorposterior length divided by the width. Statistical significance was tested using one-way ANOVA with KruskalWallace post hoc analysis. (D) Analysis of FRET intensity measurements for yellow $(y)$ control RNAi or Cdep RNAi v104438 on chromosome II, or $w$ RNAi and Cdep RNAi 31168 on chromosome III. Each dot represents one cluster measurement and statistical significance was tested using one-way ANOVA with Tukey post hoc analysis. (E) Representative maximum intensity projection of stage 10 egg chamber stained for Cdep::GFP (green) and Ecadherin (magenta). (E') Border cell cluster zoom from (E). (F) Migrating border cell cluster stained as in (E). (G) Medial view of a border cell cluster with arrows denoting border/border cell boundaries and "p" marking polar cell nuclei. (H) Zoom of epithelial follicle cells stained as (E). (I) Stage 10 egg chamber expressing RNAi (v104438) against Cdep stained as in (E). (J) Stage 10 egg chamber, (J'-J") zoom of the border cell cluster expressing LifeActGFP and UAS-2xHA::Cdep. Cell that fell off, an ectopic protrusion and abnormal protrusion morphology marked by red, orange and white arrows, respectively. All scale bars $=20 \mu \mathrm{m}$.

To determine whether Cdep like FARP2 is a RacGEF, we expressed Cdep or control RNAi for yellow ( $y$ ) or $w$ genes in combination with a validated RacFRET activity probe (Wang et al., 2010). Indeed, knockdown of Cdep with two independent RNAi constructs significantly lowered RacFRET intensities compared to the controls, indicating Cdep is a bona fide RacGEF in the border cells (Fig. 3D), in addition to the four known border cell Rac GEFs (Bianco et al., 2007; FernándezEspartero et al., 2013; Wang et al., 2018).

We found that an endogenously GFP-tagged Cdep (Cdep::GFP) is expressed in all follicle cells, including border cells (Fig. 3E-H). Costaining with the cell adhesion molecule E-cadherin, which is enriched in adherens junctions, showed Cdep localizes to basolateral membranes in border cell clusters and in the follicular epithelium (Fig. 3E-H). High resolution imaging of migrating clusters showed that Cdep is particularly enriched basally at polar/border cell membranes (Fig. 3F) and at border/border cell membranes (Fig. 3G). Like Scrib module proteins, Cdep is less abundant at border/nurse cell interfaces during migration (Fig. 3F and G). In epithelial cells, Cdep similarly shows basolateral membrane enrichment (Fig. 3H). Expression of Cdep RNAi eliminated detectable expression of Cdep::GFP from border cells (Fig. 3I, I') and epithelial follicle cells (Fig. 3I), validating the knockdown. Thus the Rac
GEF Cdep exhibits similarities to Scrib module proteins in both protein localization and knockdown phenotypes.

Rac activity is strongly associated with F-actin polymerization, protrusion, and migration in border cells (Murphy and Montell, 1996; Wang et al., 2010) and many other cell types (Ridley, 2015). To determine if overexpression of Cdep would affect border cell protrusions, we crossed c306Gal4 to UAS-hemagluttanin (2x-HA)-tagged Cdep. Clusters over-expressing HA::Cdep exhibited morphological abnormalities that included unusually long and thin protrusions from the lead cell and ectopic side protrusions (Fig. 3J and J'). Clusters also split (Fig. 3J). The overexpressed protein accumulated ectopically in the cytoplasm in addition to its normal membrane localization (fig. 3J"), demonstrating that the proper level and/or localization of Cdep is important for normal protrusion morphology and cluster cohesion. Together, these results identify Cdep as a basally localized RacGEF whose knockdown phenocopies that of Scrib module components. So we investigated the effects of apicobasal polarity proteins on Cdep localization. 

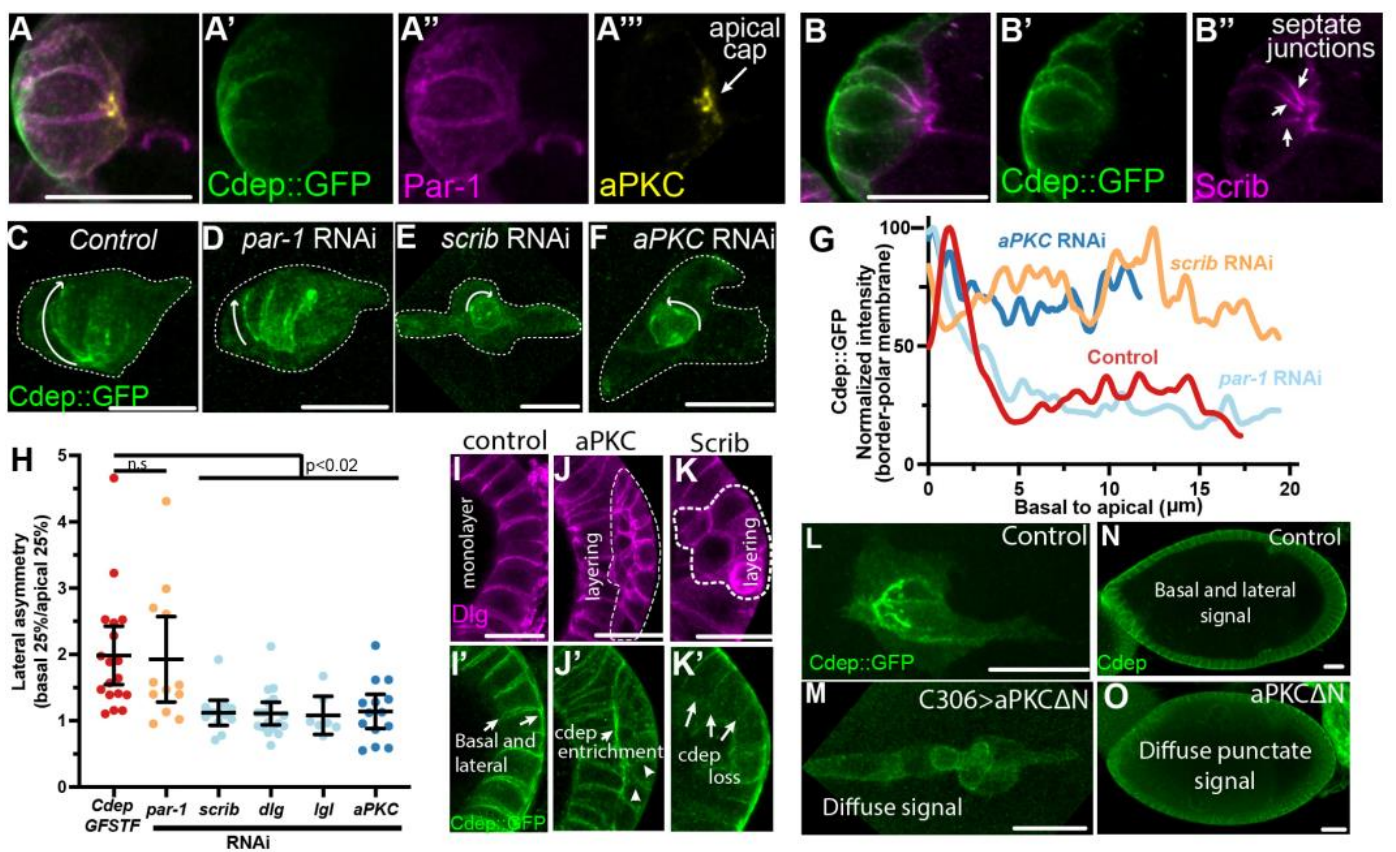

KC RNAi

Figure 4: The Scrib module and aPKC are both required for Cdep localization in border cell cluster basal membranes. (A) Overlay of border cell cluster stained for endogenously tagged Cdep::GFP (A', green), Par-1 (A", magenta), and aPKC ( $A^{\prime \prime \prime}$, yellow) marked with white arrow. (B) Overlay of border cell cluster stained for GFPCdep (B', green), and Scribble (B", magenta). (D-F) Representative images of border cell clusters expressing GFP-Cdep in combination with indicated RNAi. (G) Representative line scans along the polar/border cell boundary from basal to apical for genotypes in (D-F). (H) Dot plot of basal to apical asymmetry of Cdep at polar/border cell membrane for indicated knockdowns. Each dot is an individual border cell cluster. Statistical significance was tested using one-way ANOVA with Kruskal-Wallace post hoc analysis. (I-K') Representative images of Dlgand Cdep::GFP expression in control (I-l'), and clusters expressing aPKC (J-J') and scrib (K-K') RNAi. Layering is outlined. (L) Control and (M) UAS-aPKC $\triangle N$ expressing border cell clusters during migration. $(\mathrm{N})$ Control and $(\mathrm{O})$ UAS-aPKC $\triangle \mathrm{N}$ expressing stage 8 egg chambers stained for GFP-Cdep (green). All scale bars $=20 \mu \mathrm{m}$. either Scrib or aPKC causes multiple layers of follicle cells to form (Fig. 4I-K). Upon loss of aPKC, Cdep::GFP was found on all cell surfaces within the layering event and with enrichment between layers (Fig. 4I'-J') . Conversely, loss of Scrib led to reduced membrane accumulation of Cdep::GFP and more diffuse cytoplasmic signal (Fig. 4K'). These data suggest that the Scrib module promotes Cdep membrane association whereas aPKC antagonizes it.

A prediction then is that expression of a constitutively active form of aPKC would reduce Cdep membrane association. As expected, when we overexpressed a constitutively active form of aPKC lacking its $\mathrm{NH}_{2}$-terminus $(\mathrm{aPKC} \Delta \mathrm{N})$ (Betschinger et al., 2003), Cdep::GFP failed to associate with basal membranes and appeared more diffuse within the cytoplasm of both border cells (Fig. 4L and $\mathrm{M}$ ) and epithelial cells (Fig. 4N and O). We conclude that the Scrib module promotes membrane association while aPKC excludes Cdep from the apical domain.

\section{The Scrib module and aPKC, but not Parl, regulate Cdep localization}

To interrogate the relationship between Cdep and the polarity modules we compared Cdep localization to that of aPKC, the Scrib module, and the basal kinase Par-1. Cdep::GFP membrane localization partially overlapped with Par-1 (Fig. 4A-A'), although Cdep was concentrated even more basally than Par-1 in these cells. Cdep was undetectable in apical membranes and thus did not colocalize with aPKC in the polar cell apical cap or at apical border cell junctions (Fig. 4A-A' and A'"). Cdep and Scrib both localized to lateral membranes, but were polarized oppositely (Fig. 4B-B'): Cdep was concentrated basally (Fig. 4A and B'), while Scrib was highest in the septate junction region, and lowest near the basal surface (Fig. 1G-I, 4B'").

To test if Cdep basolateral membrane localization depends on apicobasal polarity proteins, we examined Cdep::GFP polarization in cells expressing RNAi lines against Parl, aPKC, and Scrib. Par-1 knockdown did not detectably change the localization of GFP::Cdep (Fig. 4C-D). In contrast, knockdown of either Scrib or aPKC caused a general reduction in Cdep::GFP membrane-association with more diffuse cytoplasmic signal (Fig. 4E-F). Line scans of border cell-polar cell contacts from basal to apical showed that Par-1 knockdown did not change Cdep asymmetry whereas Scrib and aPKC did (Fig. 4G and H). Dlg and Lgl knockdowns phenocopied Scrib (Fig. 4H).

Analysis of posterior epithelial follicle cells from the same egg chambers also showed a difference in Cdep membrane localization. Knockdown of

\section{membranes is sufficient to suppress Scrib phenotypes \\ Localizing Cdep to basal}

Scrib knockdown causes diverse phenotypic effects and Scrib interacts with dozens of binding partners (Bonello and Peifer, 2019), raising the question as to which effectors mediate each Scrib function. Since knockdown of Scrib disrupts Cdep membrane localization, we wondered whether localizing Cdep basolaterally might rescue any aspect of the Scrib knockdown phenotype, especially border cluster cohesion defects. We used the GrabFP system to re-localize Cdep::GFP to basal membranes. GrabFP is a nanobody-based GFP trap that was developed to relocate GFP tagged proteins to specific subcellular locations (Harmansa et al., 2017). For these experiments, we expressed UAS-scrib RNAi using c306Gal4 in the absence of tubGal80 ${ }^{\text {ts }}$, which resulted in egg chambers with severe border cell migration defects (Fig. 5A) and others with extensive anterior follicle cell layering (Fig. 5B). Under these conditions, most egg chambers eventually became necrotic (Fig. 5B). Expression of GrabFPbasal efficiently restored basolateral localization of Cdep::GFP in scrib RNAi-expressing follicle cells (Fig. 5C and D) and ameliorated multiple Scrib knockdown phenotypes, while expression of GrabFPapical, which in normal epithelia localizes to apical membranes, was less effective (Fig. 5E-H). In the egg chambers in which GrabFPapical failed to rescue layering, we noted that Cdep::GFP remained mostly diffuse in the cytoplasm (Fig. 5F). In the egg chambers in which layering was rescued, Cdep::GFP was present on both basolateral and apical membranes (Fig. 5G). Thus rescue correlated with Cdep membrane localization. In the absence of GFP::Cdep, most c306Gal4>UAS-ScribRNAi, GrabFPbasal egg chambers died, and the few that survived exhibited extensive anterior layering (Fig. $5 \mathrm{H}$ ). 
However, inclusion of Cdep::GFP allowed us to recover many more viable egg chambers, only $10 \%$ of which showed layering (Fig. 5H). The remaining 90\% exhibited some degree of border cell migration, including complete migration in $20 \%$ (Fig. $5 \mathrm{H}$ ). We conclude that basolateral membrane targeting of Cdep is sufficient to support egg chamber viability, restore follicle cell epithelial polarity, and promote border cell cluster cohesion and migration.

In further support of the sufficiency of basolateral Cdep to rescue epithelial follicle cell defects, we found that basolaterally localized Cdep also reduced the frequency of ovariole strands containing even a single egg chamber of any stage with any epithelial layering (Fig. 5I). Again, GrabFPapical had a relatively weaker effect (Fig. 5I). In addition to reducing the frequency of egg chambers with multiple follicle cell layers, basolateral re-localization of Cdep significantly reduced the number of cell layers (Fig. 5J) ( $\mathrm{p}<.0001)$, and GrabFPapical was again less effective $(\mathrm{p}=.0506)$. These findings provide evidence that a major function of the Scrib module during egg chamber development is to localize Cdep to basolateral membranes.

\section{Scrib and Cdep sculpt specialized basal protrusions between border cells}

Due to the cluster cohesion and ectopic protrusion defects caused by Scrib module and Cdep knockdowns, we examined border cell/border cell and border cell/polar cell contacts carefully and at high resolution in both wild type and RNAi-expressing cells. To clearly observe individual cell morphologies, we labeled single border cells using the FLPOUT technique. This technique highlighted that most outer, migratory cells exhibited basolateral, fanshaped protrusions that enwrapped their neighbors (Fig. 6A and $\mathrm{B}$ ), rather than extending outward between nurse cells like leading protrusions.

Given the strong association between Rac and lamellipodia, we hypothesized that the $\mathrm{Scrib} / \mathrm{Cdep} / \mathrm{Rac}$ pathway might regulate these enwrapping protrusions. Therefore, we generated single border cell clones expressing either $w$ RNAi as a control, or Scrib or Cdep RNAi together with GFP::moesin. Scrib and Cdep RNAi caused similar morphological changes to the protrusions (Fig. 6C and D), which tended to extend outwards rather than enwrapping neighbors. They were also spiky, shorter, and more numerous than in controls (Fig. 6E and F), resembling filopodia that are normally embedded within lamellipodia. Lgl and Dlg
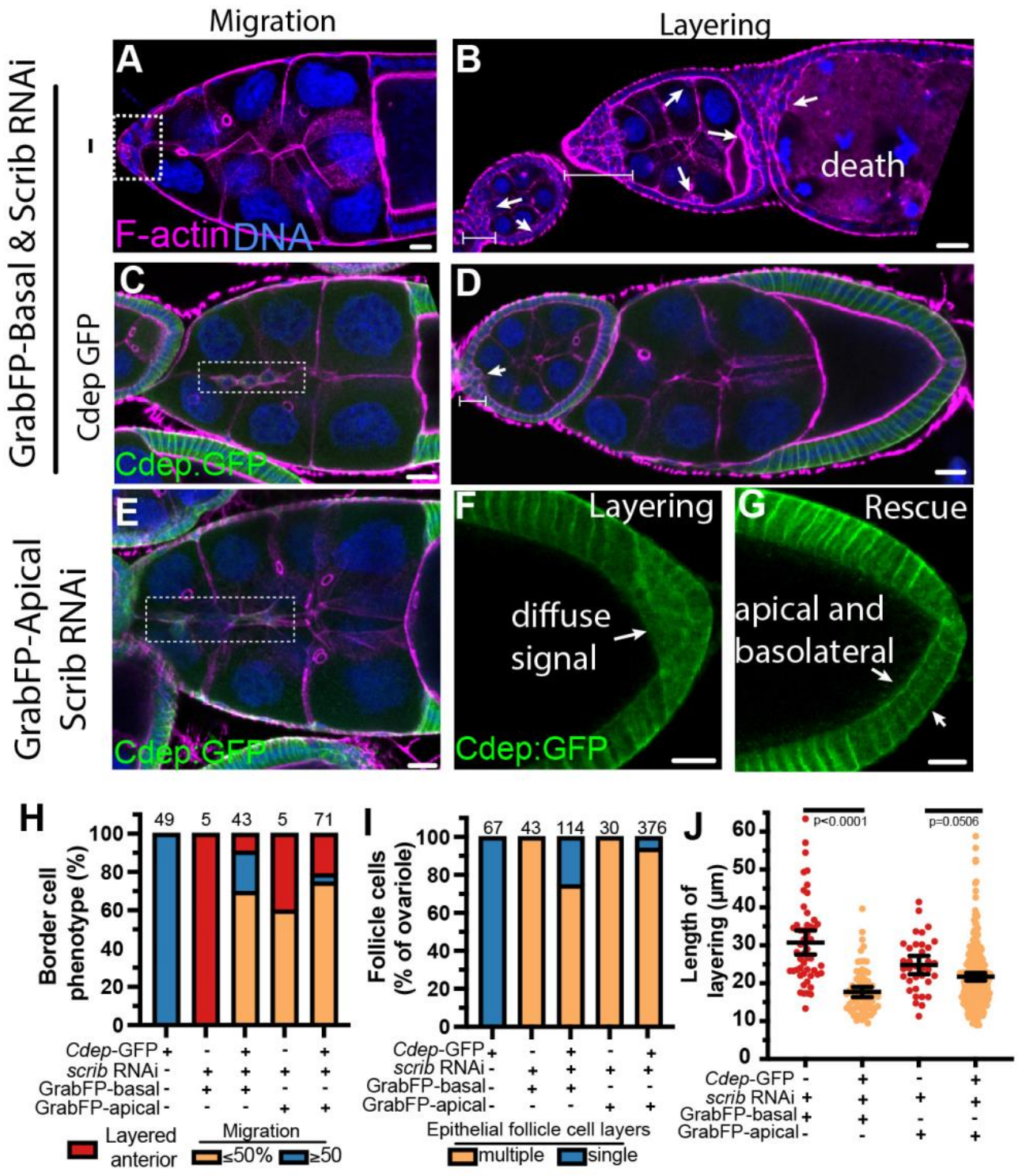

Figure 5: Re-localizing Cdep to the basal membrane suppresses border cell migration and epithelial layering caused by Scrib module knockdown. (A-B) Egg chambers expressing GrabFP-Basal and Scrib RNAi stained for F-actin with phalloidin (magenta) and DNA with hoechst (blue). Dotted box in (A) represents epithelial layering in the anterior. Arrows point to layering events throughout the egg chambe and bars represent the length measurement for anterior layering events (B). (C-D) Egg chambers coexpressing Cdep::GFP, UAS-GrabFP-basal and UAS-scrib RNAi. Dashed box in (C) represents partial rescue of border cell migration. (E) Egg chamber co-expressing Cdep::GFP, UAS-GrabFP-apical and UAS-scrib RNAi. Dashed box represents partial rescue of border cell migration. (F) Posterior layering phenotype observed in the same genotype as (E). (G) Partial rescue of posterior layering phenotype ir the same genotype as $(E)$. (H) Quantification of migration defects as $>50 \%$ of the migration path completed (blue), $<50 \%$ of the migration path (orange) or a layering replacing any definable border cell cluster (red) (I) Quantification of ovariale strands with either no layering events (blue), or any number of layering events (orange) within that strand. (J) Quantification of anterior layering size as drawn from the anterior tip of the egg chamber to the posterior-most edge of the anterior layer. Statistical significance was tested using oneway ANOVA with Kruskal-Wallace post hoc analysis. All scale bars $=20 \mu \mathrm{m}$.

knockdown caused similar effects (Fig. 6E and F). These data suggest that the Scrib module and Cdep normally sculpt basolateral enwrapping protrusions.

The enwrapping protrusions were restricted to basolateral surfaces. At the apical surface, control cells expressing w RNAi strictly respected the 
polar cell boundary and did not overlap the apical cap (Fig. 6G-G"). By contrast, cells expressing scrib (Fig. H-H”) or Dlg (Fig. 6I-I") RNAi encroached upon the polar cell apical domain, and could be seen covering it (Fig. 6H", I"). We conclude that border cells normally produce two distinct types of protrusions: spear-shaped protrusions that extend between nurse cells probing for chemoattractants and available space, and enwrapping lamellipodia that mediate interactions between cells of the migrating cluster. Apicobasal polarity proteins normally confine these protrusions to basolateral surfaces.

\section{Discussion}

\section{Specialized basal lamellipodia promote cluster cohesion}

Collective cell migration is a widespread phenomenon that drives much of embryonic development, wound healing, and tumor metastasis. In migrating collectives, there are typically leader cells that extend protrusions outward from the group to steer it. In vitro studies have shown that the Scrib and Par modules relocate to the leading edges of individually migrating cells that have undergone epithelial to mesenchymal transition (Etienne-Manneville, 2008) and in cells at the free edge of scratch wounds (Dow et al., 2007; Zegers and Friedl, 2014). There, they cooperate rather than compete, stabilizing the GEF beta-pix, thereby stimulating Cdc42 and Rac1 activities, and thus promoting protrusion. More traditional apicobasal membrane domains persist at junctions between eader cells and follower cells and between follower cells (Khalil and Friedl, 2010; Weber et al., 2012). Additionally, follower cells produce so-called cryptic basal protrusions in the direction of migration, which extend underneath the cell in front of them. These protrusions generate traction with the substrate and coordinate leading/lagging polarity amongst the cells moving collectively. Such protrusions have also been described in vivo, for example in epithelial follicle cells of early stage Drosophila egg chambers whose collective migration along the basement membrane causes the entire follicle to rotate (Barlan et al., 2017).

Here we describe basal lamellipodial protrusions that are most clearly observed when labeling a single cell within border cell clusters. These protrusions are dynamic, especially during delamination, and extend from one outer, migratory cell to enwrap a neighbor, either a polar cell or another migratory cell (Fig. 7A). These enwrapping protrusions are distinct in shape and orientation from the outward-directed protrusions that extend between nurse cells, the largest and most stable of which is produced by the leading cell. They are also distinct from classic cryptic protrusions in that they are not invariably oriented in the direction of cluster migration. In fact, the lead cell can $\mu \mathrm{m}$. hsp-flp > AyGal4
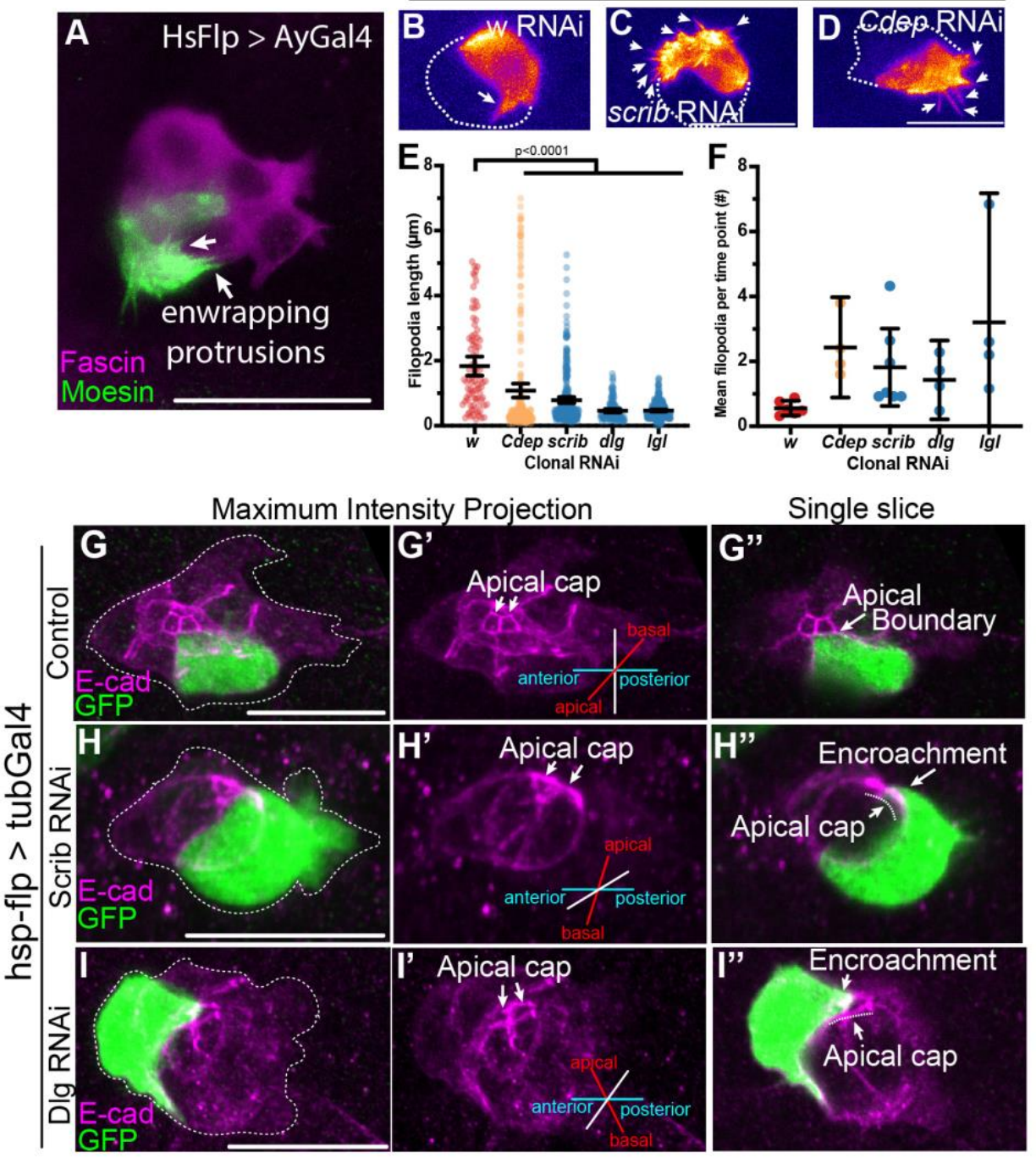

Figure 6: Cluster organization and cohesion depend on the Scrib module and Cdep. (A) Border cell cluster clonally expressing Moesin::GFP (green) under the control of hsp70>Flippase; Act5CGal4. Arrow points to an example of enwrapping protrusions. (B-D) Single time-points from a 25 min maximum intensity projection movie of single clonal border cells co-expressing UASmoesin::GFP and RNAi against white (A), scrib (B) or Cdep (C) driven by hsp70>Flippase; Act5CGal4. Arrow points to a cryptic protrusion that would not be visible in a border cell cluster uniformly expressing a fluorophore. Dashed line represents the boundary of the border cell cluster as a whole. (E) Quantification of the mean cryptic filopodial length across all time points for all samples. Each dot represents one protrusion. Statistical significance was tested using one-way ANOVA with Kruskal-Wallace post hoc analysis. (F) Quantification of the average number of filopodia per time point per genotype. Each dot represents one egg chamber. (G-I) Maximum intensity projections of border cell clusters stained for E-cadherin (magenta) and co-expressing UAS-GFP (green) and RNAi against $w\left(\mathrm{G}-\mathrm{G}^{\prime}\right)$ or scrib $\left(\mathrm{H}-\mathrm{H}^{\prime}\right)$, or dlg (l-l') driven by hsp70>Flippase; tubGal4. Dashed line represents the boundary of the border cell cluster. Moesin::GFP marks the boundary of the clonal cell. Arrows point to the apical cap of the polar cells. Distinct boundary at the apical cap in control (G") vs encroachment of the apical cap in scrib or dlg RNAi (H" and I"). All scale bars= 20

extend an enwrapping protrusion in the opposite direction to envelop the basal portion of the polar cells or another migratory cell (Fig. 6B, Fig. 7A). These enwrapping protrusions provide extensive cell-cell contacts which seem likely to enhance cluster cohesion. The relationship between enwrapping protrusions and cryptic protrusions will be interesting to decipher. It is possible that cryptic protrusions become enwrapping protrusions when the collective moves as a cluster within a relatively 

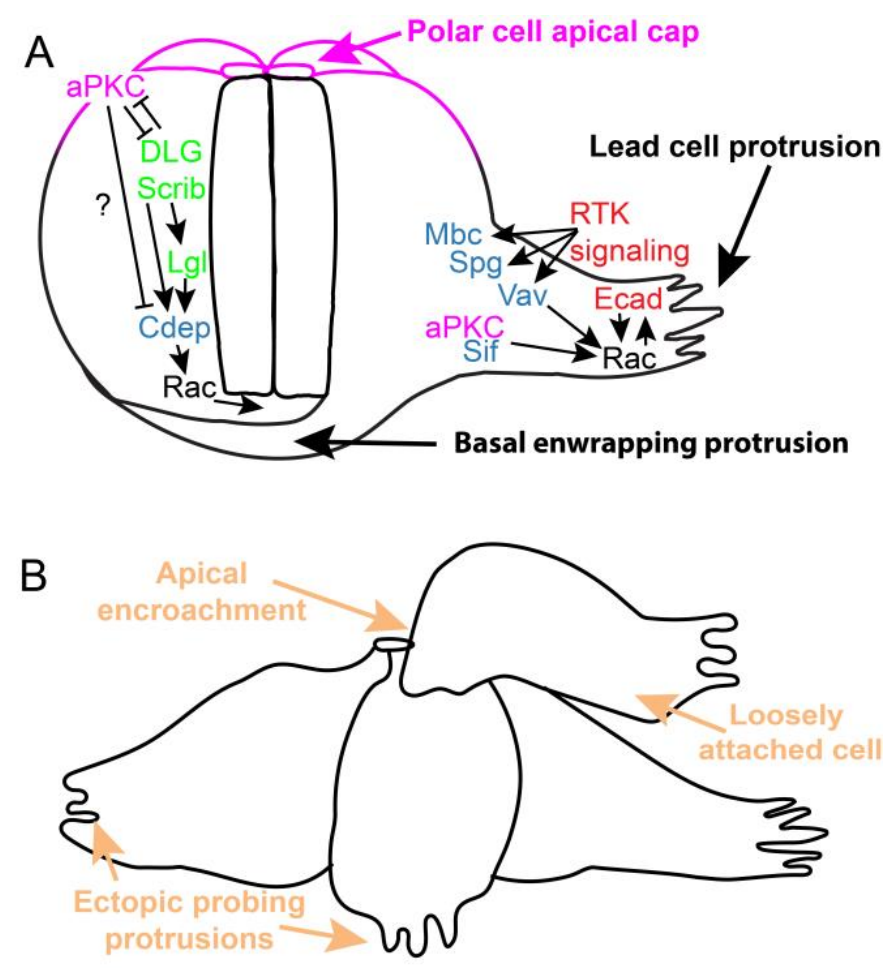

Scrib module or Cdep KD depolarizes protrusions and destabilizes cluster cohesion

Figure 7: Model of polarity dependent Cdep localization and function in border cell cohesion through basal enwrapping protrusions. (A) Illustration of wildtype border cell cluster overlaid with models of known effectors of Rac signaling in lead cell protrusions and in polarity dependent basal enwrapping protrusions. (B) Illustration border cell clusters lacking Scrib module or Cdep expression contain loosely attached cells, outwardly directed ectopic protrusions and apical cap encroachment.

compliant, 3D, cell-rich tissue, rather than as an epithelial sheet migrating on a stiff, flat extracellular matrix substrate.

\section{Scrib, Cdep, and Rac promote cluster cohesion}

Scrib, Cdep, and Rac appear to function in a common pathway to regulate basal lamellipodia. The evidence that the Scrib module and Cdep function in a common pathway includes similarities in localization and knockdown phenotypes. Knockdown of either Scrib or Cdep produces similarly elongated clusters, ectopic probing protrusions, and cluster splitting, indicative of disrupted cluster cohesion (Fig. 7B). Cdep membrane localization is disrupted in Scrib module knockdowns, placing Cdep downstream. The most compelling evidence for the Scrib-Cdep relationship is that basolateral membrane targeting of Cdep is sufficient to suppress Scrib knockdown phenotypes.

The evidence that Cdep is a RacGEF is that Rac activity is reduced upon Cdep knockdown. This effect is all the more remarkable considering that several other GEFs also activate Rac in border cells: Mbc (Dock180) and Spg (Bianco et al., 2007), Vav (Fernández-Espartero et al., 2013), and Sif (Tiam1) (Wang et al., 2018) activate Rac in leading border cell protrusions where Rac activity is slightly higher in than in the rest of the cluster (Wang et al., 2010; Fernández-Espartero et al., 2013). Though it is clear that there is active Rac throughout the whole cluster, its function outside of lead protrusions has not been explored. Cdep may make a minor contribution to Rac activity in lead protrusions; however its main effect seems to be in promoting cluster cohesion. Taken together these results support the model shown in Fig. 7A.

\section{Regulation of Cdep by apicobasal polarity proteins}

In addition to positive regulation by the Scrib module, Cdep is negatively regulated by aPKC because constitutive aPKC is sufficient to evict Cdep from the membrane. This effect could either be a direct effect of aPKC on Cdep or an indirect effect via Lgl or both. Interactions amongst polarity proteins and Rho GTPases are notoriously complex, including redundancy and multiple feedback loops (Mack and Georgiou, 2014). Polarity signaling complexes and Rho GTPase activities appear to be well-conserved between border cells and mammalian cells that migrate collectively, and a basal Rac GEF dependent upon Scribble has even been predicted (Zegers and Friedl, 2014). The results presented here suggest that FARP2 would be an excellent candidate in collectively migrating mammalian cells. FARP2 has not been extensively studied, however the little that has been reported is intriguing. Colorectal carcinomas invade and spread as small groups of cells with apicobasal polarity, and FARP2 was identified in a screen for Rho family GEFs that are required for collective invasion (Libanje et al., 2019). It will be interesting to determine whether it serves a similar function downstream of Scrib signaling activities in that context.

The relationship between Cdep and Scrib may also be complex. It is noteworthy that Scrib accumulates to the highest level just basal to the adherens junction whereas Cdep accumulates basally. The two proteins overlap in lateral membranes but it is possible that high levels of Scrib antagonize Cdep and/or vice versa to generate the opposing localization gradients.

\section{Cdep is a novel Scrib module effector for epithelial polarity}

The most surprising result presented here is that basolateral membrane targeting of Cdep is sufficient to rescue apicobasal polarity in the follicular epithelium when Scrib is knocked down. A trivial explanation would be that somehow the GrabFP reduces the effectiveness of Scrib RNAi, for example by stabilizing Scrib protein. We ruled that out however because Scrib protein is still absent from the rescued cells. The most likely explanation for this dramatic beneficial effect is that basolateral Cdep feeds back into the cell polarity circuit, either by participating as a scaffolding protein for Dlg or Lgl, or through Rac activation. For example, one study has implicated Rac and the RacGTPbinding protein Pak in apicobasal polarity in border cells (Felix et al., 2015), and there is ample evidence for interdependence between the core polarity modules and Rho family GTPases as mentioned above (Mack and Georgiou, 2014). Given the dozens of Scrib module binding proteins and the complexity and redundancies amongst Rho family GEFs, it is truly remarkable that membrane tethering of Cdep alone is sufficient to substantially rescue epithelial polarity.

\section{Materials and Methods}

\section{Drosophila husbandry and genetics}

A detailed list of all fly strains used in this study, their source and the figures in which they were used is documented in Table 1. Strains outside of lab stocks were ordered from the Bloomington Drosophila Stock Center, the Vienna Drosophila Resource Center, or kindly donated (see table 1). Most crosses were grown at $25^{\circ} \mathrm{C}$ and flies were fattened by yeast addition on an ad libitum basis for three days at $29^{\circ} \mathrm{C}$ before dissection. The exceptions were for crosses containing the conditional expression construct, tubGal80ts, in which flies were grown at $18^{\circ} \mathrm{C}$ and transitioned to $30^{\circ} \mathrm{C}$ for fattening. tubGal80ts is a temperature dependent repressor of Gal4 
activity, thus at restrictive temperature $\left(18^{\circ} \mathrm{C}\right)$ Gal4 remains inactive and at permissive temperature $\left(30^{\circ} \mathrm{C}\right)$, Gal80ts is inactive therefore allowing Gal 4 to be active. Experiments that required clonal border cells expressing RNAi and a fluorescent clonal marker were generated using the FLP/FRT clonal marking system. RNAi stocks were crossed to either hsp70-FLP; Acty17bGal4, UAS-moesin-GFP or hsp70-FLP; Tub[FRT]CD2[FRT]Gal4, UAS-GFP/SM5a were heat-shocked two times at $37^{\circ} \mathrm{C}$ with at least 6 hours between each heat shock. Flies were fattened for $48 \mathrm{hr}$ and dissected as stated below for fixed staining or live imaging.

Table 1. Fly strains used in this study.

\begin{tabular}{|c|c|c|c|}
\hline Fly strain & Source & Figures & Notes \\
\hline$W^{1118} ;+;+$ & Lab stock & $\begin{array}{l}1 \mathrm{~A}, \mathrm{~B}, \\
\mathrm{C}, \mathrm{H}, \mathrm{I}, \mathrm{J}\end{array}$ & \\
\hline $\begin{array}{l}W^{1118 ;} \\
\text { slbo>LifeActGFP/CyO; + }\end{array}$ & Lab stock & $1 D-G$ & \\
\hline $\begin{array}{l}\text { c306Gal4; } \\
\text { slbo>LifeActGFP, } \\
\text { tubGal80's/CyO; +/+ }\end{array}$ & Lab stock & 2 (all) & \\
\hline $\mathrm{y}^{1}, \mathrm{v}^{1} ;+;$ UAS-white RNAi & $\begin{array}{l}\text { BDSC* }^{*} \\
(\# 33623)\end{array}$ & $\begin{array}{l}2 A, B, \\
C, D, ~ I, \\
\text { J, L, P, } \\
3 A, \quad C, \\
D, \quad 6 B, \\
E, F, G\end{array}$ & TRiP.HMS00017 \\
\hline$W^{1118} ;+;$ UAS-scrib RNAi & $\begin{array}{l}\text { BDSC } \\
(\# 59080)\end{array}$ & $\begin{array}{l}2 A^{\prime}, \mathrm{E}, \mathrm{I}, \\
\mathrm{J}, \mathrm{K}, \mathrm{M}, \\
\mathrm{P}, \quad 4 \mathrm{E}, \\
\mathrm{G}, \mathrm{H}, \mathrm{K}, \\
5 \text { (all), } \\
6 \mathrm{C}, \mathrm{E}, \\
\mathrm{F}, \mathrm{H}\end{array}$ & $\begin{array}{l}\text { Deposited by } \mathrm{H} \text {. } \\
\text { Richardson }\end{array}$ \\
\hline $\mathrm{y}^{1}, \mathrm{~V}^{1} ;++$ UAS-scrib RNAi & $\begin{array}{l}\text { BDSC } \\
(\# 29552)\end{array}$ & 21 & TRiP.JF03229 \\
\hline$w ;++$ UAS-dlg RNAi & $\begin{array}{l}\text { VDRC** } \\
(\# 41136)\end{array}$ & $\begin{array}{l}2 \mathrm{~B}^{\prime}, \mathrm{F}, \mathrm{I}, \\
\mathrm{J}, \mathrm{N}, \mathrm{P},\end{array}$ & $\begin{array}{l}\text { Construct ID: } \\
\text { GD } 4689\end{array}$ \\
\hline $\begin{array}{l}y^{1}, s c^{*}, v^{1}, \operatorname{sev}^{21} ;+ \text { UAS- } \\
\text { dlg RNAi }\end{array}$ & $\begin{array}{l}\text { BDSC } \\
(\# 31181)\end{array}$ & 21 & TRiP.JF01694 \\
\hline$w ;+;$ UAS-lg/ RNAi & $\begin{array}{l}\text { VDRC } \\
(\# 51249)\end{array}$ & $\begin{array}{l}2 C^{\prime}, \mathrm{G}, \\
\mathrm{l}, \mathrm{j}, \mathrm{O}, \\
\mathrm{P}, 4 \mathrm{H}\end{array}$ & $\begin{array}{l}\text { Construct ID: } \\
\text { GD } 4047\end{array}$ \\
\hline $\begin{array}{l}y^{1}, s C^{*}, v^{1}, \operatorname{sev}^{21} ;+ \text { UAS-lgl } \\
\text { RNAi }\end{array}$ & $\begin{array}{l}\text { BDSC } \\
(\# 35773)\end{array}$ & 21 & TRiP.HMS01522 \\
\hline $\mathrm{y}^{1} \mathrm{w} ;+;$ GFSTF-Cdep & $\begin{array}{l}\text { BDSC } \\
(\# 61789)\end{array}$ & $\begin{array}{l}\text { 3E, } \quad F \text {, } \\
\mathrm{G}, \mathrm{H}, \mathrm{I}\end{array}$ & $\begin{array}{l}\text { MI06830- } \\
\text { GFSTF.1, } \\
\text { Deposited by S. } \\
\text { Parkhurst }\end{array}$ \\
\hline $\begin{array}{l}\text { c306Gal4; } \\
\text { LifeActGFP/CyO; +/+ }\end{array}$ & Lab stock & $3 \mathrm{~A}, \mathrm{~B}, \mathrm{C}$ & \\
\hline w; UAS-Cdep RNAi; + & $\begin{array}{l}\text { VDRC } \\
(\# 104438)\end{array}$ & $\begin{array}{l}3 \mathrm{~B}, \mathrm{C}, \\
\mathrm{D}, \mathrm{I}, 6 \mathrm{D} \\
\mathrm{E}, \mathrm{F}\end{array}$ & $\begin{array}{l}\text { Construct ID: } \\
\text { KK } 108821\end{array}$ \\
\hline w*; UAS-Rac-Fret; + & Lab stock & $3 D$ & \\
\hline
\end{tabular}

\begin{tabular}{|c|c|c|c|}
\hline $\mathrm{w}^{*} ;++$ UAS-Rac-Fret & Lab stock & 3D & \\
\hline $\begin{array}{l}y^{1}, \quad s c^{*}, v^{1}, \text { sev }^{21} ; \text { UAS- } \\
\text { yellow RNAi; + }\end{array}$ & $\begin{array}{l}\text { BDSC } \\
(\# 64527)\end{array}$ & $3 \mathrm{D}$ & TRiP.HMC05546 \\
\hline $\mathrm{y}^{1}, \mathrm{~V}^{1} ;++$ UAS-Cdep RNAi & $\begin{array}{l}\text { BDSC } \\
(\# 31168)\end{array}$ & $3 D$ & TRiP.JF01661 \\
\hline $\begin{array}{l}W^{1118} ;+; \quad \text { UAS-2xHA- } \\
\text { CdepE/F }\end{array}$ & $\begin{array}{l}\text { Made for } \\
\text { this study }\end{array}$ & $3 \mathrm{~J}$ & \\
\hline $\begin{array}{l}\text { c306Gal4; }+; \quad \text { GFSTF- } \\
\text { Cdep }\end{array}$ & $\begin{array}{l}\text { Combined } \\
\text { for this } \\
\text { study }\end{array}$ & $\begin{array}{l}4 \mathrm{C}, \mathrm{D}, \\
\mathrm{E}, \mathrm{F}, \mathrm{G}, \\
\mathrm{H}, \mathrm{I}, \mathrm{J}, \\
\mathrm{K}, \mathrm{L}, \mathrm{M}, \\
\mathrm{N}, \mathrm{O}, 5 \\
\text { (all) }\end{array}$ & \\
\hline UAS Par1 RNAi & $\begin{array}{l}\text { VDRC } \\
(\# 52556)\end{array}$ & $\underset{H}{4 D}, \quad G$, & $\begin{array}{l}\text { Construct ID: } \\
\text { GD } 14836\end{array}$ \\
\hline UAS aPKC RNAi & $\begin{array}{l}\text { VDRC } \\
(\# 105624)\end{array}$ & $\underset{H, J}{4 F}, \quad G$, & $\begin{array}{l}\text { Construct ID: } \\
\text { KK } 100874\end{array}$ \\
\hline$w ;+;$ UAS-aPKCdeltaN & $\begin{array}{l}\text { BDSC } \\
(\# 51673)\end{array}$ & $4 \mathrm{M}, \mathrm{O}$ & $\begin{array}{l}\text { Deposited by } \mathrm{H} \text {. } \\
\text { Bellen }\end{array}$ \\
\hline $\begin{array}{l}\mathrm{w}^{*} ; \quad \mathrm{Kr} / \mathrm{H} / \mathrm{CyO} ; \text { UAS- } \\
\text { GrabFP.Basal }\end{array}$ & $\begin{array}{l}\text { BDSC } \\
(\# 68175)\end{array}$ & 5 (all) & $\begin{array}{l}\text { Deposited by } M \text {. } \\
\text { Affolter }\end{array}$ \\
\hline $\begin{array}{l}\mathrm{w}^{*} ; \quad \mathrm{Kr} \mathrm{rr}^{\mathrm{H}} / \mathrm{CyO} ; \quad \text { UAS- } \\
\text { GrabFP.Apical }\end{array}$ & $\begin{array}{l}\text { BDSC } \\
(\# 68178)\end{array}$ & $\begin{array}{l}\text { 5E, } \mathrm{F}, \\
\mathrm{G}, \mathrm{H}, \mathrm{I},\end{array}$ & $\begin{array}{l}\text { Deposited by } M \text {. } \\
\text { Affolter }\end{array}$ \\
\hline $\begin{array}{l}\text { hsp70-FLP; } \\
\text { Acty17bGal4, } \\
\text { moesin-GFP }\end{array}$ & Lab stock & $\begin{array}{l}6 \mathrm{~A}, \mathrm{~B} \\
\mathrm{C}, \mathrm{D}, \mathrm{E} \\
\mathrm{F}\end{array}$ & \\
\hline $\begin{array}{l}\text { hsp70-FLP; } \\
\text { Tub[FRT]CD2[FRT]Gal4, } \\
\text { UAS-GFP/SM5a }\end{array}$ & $\begin{array}{ll}\text { Gift } & \text { from } \\
\text { AM } & \text { Pret } \\
\text { Lab } & \end{array}$ & $6 \mathrm{G}, \mathrm{H}, \mathrm{I}$ & \\
\hline
\end{tabular}

Drosophila Resource Center.

\section{Immunohistochemistry}

5-7 fattened adult female flies were dissected in Schneider's Medium (ThermoFisher, catalog \#21720001) supplemented with $20 \%$ fetal bovine serum and $1 x$ antimycotic/antibiotic (VWR, catalog \#45000-616). Ovarioles were separated from the muscle sheath using Dumont style 5 stainless steel forceps (Electron Microscopy Sciences) before fixation. Egg chambers were fixed in $4 \%$ paraformaldehyde in phosphate buffered saline (PBS) for a total of $15 \mathrm{~min}$ at room temperature before washing three times 15 min with PBS $+0.2 \%$ TritonX-100 (PBST). Primary antibodies were diluted in PBST and ovarioles were incubated overnight at $4^{\circ} \mathrm{C}$. Egg chambers were then washed with PBST three times at room temperature, incubated in fluorescently conjugated secondary antibodies (Invitrogen, all AlexaFluor conjugated goat antibodies) in PBST for $2 \mathrm{hr}$, and washed three times more with PBST. Samples were then mounted in VectaShield (Vector Laboratories, catalog $\# \mathrm{H}-1000$ ) mounting medium and stored at $4^{\circ} \mathrm{C}$ until imaging. Egg chambers were mounted on a \#1.5 coverglass and imaged with either a Zeiss LSM780 or LSM800 confocal microscope using either a PlanAPO 20x 1.2NA or a PlanAPO 40x 1.4NA objective. All image settings were kept exactly the same within each experiment.

Antibodies used in this study include: rabbit anti-Scrib (kind gift from $\mathrm{Dr}$. Chris Doe, 1:2000), guinea pig anti-Scrib (kind gift from Dr. David Bilder, 1:500), Rabbit anti-Par-1 (a kind gift from Dr. Jocelyn McDonald, 1:500), mouse anti-PKC $\zeta$ (sc-216) and rabbit anti-PKC $\zeta$ (sc-17781), mouse anti- 
HA (sc-7392, 1:1000), and rabbit anti-LGL (sc-9826) were from Santa Cruz Biotechnology and used at 1:500, mouse anti-Dlg (4F3,1:25), mouse antiCut (2B10, 1:100) and rat anti-Ecad (DEcad, 1:20) were from the Developmental Studies Hybridoma Bank, rabbit anti-GFP ( A11122, $1: 1000)$ from ThermoFisher, chicken anti-GFP (ab13970, 1:1000) from Abcam. Lectin PNA Alexa 647 was purchased from Thermo Fisher Scientific (L32460).

\section{Live imaging}

Egg chambers were dissected from 5-7 fattened adult female flies in Schneider's Medium supplemented with $20 \%$ fetal bovine serum and $1 x$ antimycotic/antibiotic. Egg chambers were washed two times with dissecting medium and mounted on Lumox imaging dishes in medium containing $0.4 \mathrm{mg} / \mathrm{mL}$ bovine insulin and 1\% low melt agarose. Egg chambers were imaged by confocal as above.

\section{Generating UAS-HA-Cdep}

The RNeasy kit (Qiagen, catalog \#74124) was used to isolate total RNA from the ovaries of six fattened female flies. RNA was converted to CDNA using the SuperScriptllI-First strand synthesis kit (ThermoFisher, catalog \#18080-51). The following primers with overhangs for subsequent Infusion cloning into the pJFRC7 vector and encoding a N-terminal 2xHA tagg were used to amplify full length Cdep isoforms E/F. Forward:GCGGCCGCGGCTCGAGATGTACCCATACGATGTTCCAGAT TACGCTGGATATCCATATGATGTTCCAGATTATGCTGGAATGTCCCT GGCCGACATGGG.

Reverse:CATGCTGTGTTGGGCAACTAATCTAGAGGATCTTTGT.

Amplicons of tagged-Cdep were isolated by gel extraction after PCR amplification using Phusion high fidelity polymerase (New England Biolabs, catalog \#E0553L). The amplicons were then cloned into the pJFRC7 5x UAS vector (ref) using Infusion cloning kit (Takara Bio, catalog \#638948). Sequenced clones were sent to BestGene for injection and stock generation.

\section{Image processing and quantification.}

Quantification of apical-basal polarization of proteins along the border cellpolar cell boundary (Fig 1H/I, Fig 3D/E): When possible the apical cap of the polar cells was identified by either E-cadherin, Phalloidin/F-actin or aPKC staining. Using ImageJ a line scan with a thickness of $.75 \mu \mathrm{m}$ was drawn tracing the border cell-polar cell boundary using the F-actin channel as a guide. Measurements were then taken in the Cdep channel and averaged over $0.50 \mu \mathrm{m}$ to smooth the signal and then normalized to the peak value within an individual border cell cluster. Representative line scans were chosen for the line graphs. To compare population differences we took the average of the $25 \%$ most basal signal divided by the $25 \%$ most apical signal for each sample. This ratiometric measurement of basal asymmetry was used for statistics and population level plotting. In a subset of polarity complex knockdown samples the apical cap could not be easily distinguished and an arbitrary pole was chosen as basal. In this condition, there was no bias seen in Cdep asymmetry regardless of orientation or start point chosen.

Quantification cluster aspect ratio (Fig 2G): A bounding box was manually drawn in FIJl around the border cell cluster in an anterior-posterior orientation. The aspect ratio of the bounding rectangle was then measured and plotted for each condition.

Quantification of cluster speed (Fig 2L): The center of mass of the border cell cluster was manually identified in each time point. The MTrackJ plugin was then used to calculate the velocity of the border cell cluster.

Quantification of Rac FRET (Fig 3D): FRET images were obtained during live cell imaging on a Zeiss LSM 780. A 458-nm laser was used for excitation of the sample. CFP and YFP images were collected simultaneously using channel 1 (464-502 nm) and channel 2 (517-570 nm) under a $40 \times / 1.1$ numerical aperture (NA) water immersion objective LD C-Apo lens. Images were taken at 16 -bits with a frame size of $512 \times$ 512 in the center of mass of the border cell cluster. CFP and YFP images were then processed using Fiji image analysis software as described before (Kardash ref). The final FRET image of the YFP/CFP ratio image was generated in ImageJ. The average FRET signal of the entire cluster was measured. All samples were observed after detachment and prior to docking.
Quantification of layering (Fig 5D/E): Images were taken of whole ovariale strands at a low zoom-level on a 20x objective using a Zeiss LSM 800 or Zeiss LSM 780. Images were 30-36 microns in thickness with a Z-step of 2.5-3 microns. Images were then manually quantified in ImageJ by observing any location within the follicular epithelium that showed layering in any stage egg chamber. Quantification of layering was done only on anterior layering events in stage eight and nine egg chambers. The distance from the anterior tip of the egg chamber to the posterior-most cell within the layering event was measured to quantify the size of the layering event.

Quantification of cryptic protrusions (Fig6 D/E): Filopodia were manually marked at the distal tip and at where the protrusion meets the circumference of the border cell cluster. The distance between the $X Y$ coordinates at the tip vs base was then quantified with the distance formula: distance $\left.=\operatorname{sqrt}\left(X_{2}-X_{1}\right)^{2}+\left(Y_{2}-Y_{1}\right)^{2}\right)$.

\section{Graphing and statistical analyses}

All data were analyzed in Excel (Microsoft). Graphs and statistics were generated in Prism 9 (GraphPad). For statistical analysis by one-way ANOVA, Bartlett's Test was run to determine if significant differences in sample deviations warranted non-parametric analysis. In cases where deviations were not significantly different, parametric ANOVA was performed with Tukey post-hoc tests of significance. In cases where deviations were found to be significantly different, non-parametric ANOVA was run with Kruskal-Wallace post-hoc tests of significance.

\section{Author Contributions}

Conceptualization, J.P.C., J.A.M, and D.J.M.; Methodology J.P.C., J.A.M, and D.J.M.; Validation, J.P.C. and J.A.M; Formal Analysis, J.P.C. and J.A.M; Investigation, J.P.C. and J.A.M; Resources , J.P.C., J.A.M, and D.J.M.; Data Curation, J.P.C. and J.A.M; Writing - Original Draft, J.P.C., J.A.M, and D.J.M.; Writing - Review \& Editing , J.P.C., J.A.M, and D.J.M.; Visualization, J.P.C. and J.A.M; Supervision, J.P.C., J.A.M, and D.J.M.; Funding Acquisition, J.P.C.and D.J.M.

\section{Acknowledgments}

We would like to acknowledge Drs. Abhinava Mishra, Lauren Penfield, Wei Dai Guangxia Miao, and Allsion Gabbert for thoughtful discussions during the course of this work. We kindly thank Dr. David Bilder and Dr. Christopher Doe for Drosophila Scrib antibody and Dr. Jocelyn McDonald for Par-1 antibody. We acknowledge the Bloomington Drosophila Stock Center and Vienna Drosophila Resource Center for fly stocks. This work was funded by an American Cancer Society award PF17-024-01-CSM to J.P.C and an National Institutes of Health award GM46425 to D.J.M.

\section{References}

Barlan, K., Cetera, M., and Horne-Badovinac, S. (2017). Fat2 and lar define a basally localized planar signaling system controlling collective cell migration. Dev. Cell 40, 467-477.e5.

Betschinger, J., Mechtler, K., and Knoblich, J.A. (2003). The Par complex directs asymmetric cell division by phosphorylating the cytoskeletal protein Lgl. Nature 422, 326-330. 
Bianco, A., Poukkula, M., Cliffe, A., Mathieu, J., Luque, C.M., Fulga, T.A., and Rørth, P. (2007). Two distinct modes of guidance signalling during collective migration of border cells. Nature 448, 362-365.

Bilder, D., Li, M., and Perrimon, N. (2000). Cooperative regulation of cell polarity and growth by Drosophila tumor suppressors. Science 289 , 113-116.

Bilder, D., Schober, M., and Perrimon, N. (2003). Integrated activity of PDZ protein complexes regulates epithelial polarity. Nat. Cell Biol. 5, 5358.

Bonello, T.T., and Peifer, M. (2019). Scribble: A master scaffold in polarity, adhesion, synaptogenesis, and proliferation. J. Cell Biol. 218, 742-756.

Bonello, T., Aguilar-Aragon, M., Tournier, A., and Thompson, B.J. (2021). A picket fence function for adherens junctions in epithelial cell polarity. Cells Dev. 203719.

Campanale, J.P., Sun, T.Y., and Montell, D.J. (2017). Development and dynamics of cell polarity at a glance. J. Cell Sci. 130, 1201-1207.

Dai, W., Guo, X., Cao, Y., Mondo, J.A., Campanale, J.P., Montell, B.J., Burrous, H., Streichan, S., Gov, N., Rappel, W.-J., et al. (2020). Tissue topography steers migrating Drosophila border cells. Science 370, 987-990.

Danelon, V., Goldner, R., Martinez, E., Gokhman, I., Wang, K., Yaron, A., and Tran, T.S. (2020). Modular and Distinct Plexin-A4/FARP2/Rac1 Signaling Controls Dendrite Morphogenesis. J. Neurosci. 40, 5413-5430.

Dow, L.E., Brumby, A.M., Muratore, R., Coombe, M.L., Sedelies, K.A., Trapani, J.A., Russell, S.M., Richardson, H.E., and Humbert, P.O. (2003). hScrib is a functional homologue of the Drosophila tumour suppressor Scribble. Oncogene 22, 92259230.

Dow, L.E., Kauffman, J.S., Caddy, J., Zarbalis, K., Peterson, A.S., Jane, S.M., Russell, S.M., and Humbert, P.O. (2007). The tumour-suppressor Scribble dictates cell polarity during directed epithelial migration: regulation of Rho GTPase recruitment to the leading edge. Oncogene 26, 2272-2282.
Etienne-Manneville, S. (2008). Polarity proteins in migration and invasion. Oncogene 27, 6970-6980.

Felix, M., Chayengia, M., Ghosh, R., Sharma, A., and Prasad, M. (2015). Pak3 regulates apical-basal polarity in migrating border cells during Drosophila oogenesis. Development 142, 3692-3703.

Fernández-Espartero, C.H., Ramel, D., Farago, M., Malartre, M., Luque, C.M., Limanovich, S., Katzav, S., Emery, G., and Martín-Bermudo, M.D. (2013). GTP exchange factor Vav regulates guided cell migration by coupling guidance receptor signalling to local Rac activation. J. Cell Sci. 126, 22852293.

Friedl, P., and Gilmour, D. (2009). Collective cell migration in morphogenesis, regeneration and cancer. Nat. Rev. Mol. Cell Biol. 10, 445-457.

Friedl, P., Sahai, E., Weiss, S., and Yamada, K.M. (2012). New dimensions in cell migration. Nat. Rev. Mol. Cell Biol. 13, 743-747.

Goldstein, B., and Macara, I.G. (2007). The PAR proteins: fundamental players in animal cell polarization. Dev. Cell 13, 609-622.

Goode, S., and Perrimon, N. (1997). Inhibition of patterned cell shape change and cell invasion by Discs large during Drosophila oogenesis. Genes Dev. 11, 2532-2544.

Goode, S., Wei, J., and Kishore, S. (2005). Novel spatiotemporal patterns of epithelial tumor invasion in Drosophila discs large egg chambers. Dev. Dyn. 232, 855-864.

Halaoui, R., and McCaffrey, L. (2015). Rewiring cell polarity signaling in cancer. Oncogene 34, 939950.

Hariharan, I.K., and Bilder, D. (2006). Regulation of imaginal disc growth by tumor-suppressor genes in Drosophila. Annu. Rev. Genet. 40, 335-361.

Harmansa, S., Alborelli, I., Bieli, D., Caussinus, E., and Affolter, M. (2017). A nanobody-based toolset to investigate the role of protein localization and dispersal in Drosophila. ELife 6.

Khalil, A.A., and Friedl, P. (2010). Determinants of leader cells in collective cell migration. Integr Biol (Camb) 2, 568-574.

Khoury, M.J., and Bilder, D. (2020). Distinct activities of Scrib module proteins organize epithelial polarity. Proc Natl Acad Sci USA 117, 1153111540. 
Knoblich, J.A. (2000). Epithelial polarity: the ins and outs of the fly epidermis. Curr. Biol. 10, R791-4.

Krahn, M.P., Bückers, J., Kastrup, L., and Wodarz, A. (2010). Formation of a Bazooka-Stardust complex is essential for plasma membrane polarity in epithelia. J. Cell Biol. 190, 751-760.

Lamouille, S., Xu, J., and Derynck, R. (2014). Molecular mechanisms of epithelial-mesenchymal transition. Nat. Rev. Mol. Cell Biol. 15, 178-196.

Lee, M., and Vasioukhin, V. (2008). Cell polarity and cancer--cell and tissue polarity as a non-canonical tumor suppressor. J. Cell Sci. 121, 1141-1150.

Libanje, F., Raingeaud, J., Luan, R., Thomas, Z., Zajac, O., Veiga, J., Marisa, L., Adam, J., Boige, V., Malka, D., et al. (2019). ROCK2 inhibition triggers the collective invasion of colorectal adenocarcinomas. EMBO J. 38, e99299.

Li, Q., Feng, S., Yu, L., Zhao, G., and Li, M. (2011). Requirements of $\mathrm{Lgl}$ in cell differentiation and motility during Drosophila ovarian follicular epithelium morphogenesis. Fly (Austin) 5, 81-87.

Macara, I.G., and McCaffrey, L. (2013). Cell polarity in morphogenesis and metastasis. Philos. Trans. R. Soc. Lond. B Biol. Sci. 368, 20130012.

Mack, N.A., and Georgiou, M. (2014). The interdependence of the Rho GTPases and apicobasal cell polarity. Small GTPases 5, 10.

Manseau, L., Baradaran, A., Brower, D., Budhu, A., Elefant, F., Phan, H., Philp, A.V., Yang, M., Glover, D., Kaiser, K., et al. (1997). GAL4 enhancer traps expressed in the embryo, larval brain, imaginal discs, and ovary of drosophila. Developmental Dynamics.

Martin-Belmonte, F., and Mostov, K. (2008). Regulation of cell polarity during epithelial morphogenesis. Curr. Opin. Cell Biol. 20, 227234.

Miao, G., Godt, D., and Montell, D.J. (2020). Integration of Migratory Cells into a New Site In Vivo Requires Channel-Independent Functions of Innexins on Microtubules. Dev. Cell 54, 501515.e9.

Mishra, A.K., Campanale, J.P., Mondo, J.A., and Montell, D.J. (2019a). Cell interactions in collective cell migration. Development 146.

Mishra, A.K., Mondo, J.A., Campanale, J.P., and Montell, D.J. (2019b). Coordination of protrusion dynamics within and between collectively migrating border cells by myosin II. Mol. Biol. Cell 30, 2490-2502.

Mlechkovich, G., Peng, S.-S., Shacham, V., Martinez, E., Gokhman, I., Minis, A., Tran, T.S., and Yaron, A. (2014). Distinct cytoplasmic domains in PlexinA4 mediate diverse responses to semaphorin 3A in developing mammalian neurons. Sci. Signal. 7, ra24.

Montell, D.J., Rorth, P., and Spradling, A.C. (1992). slow border cells, a locus required for a developmentally regulated cell migration during oogenesis, encodes Drosophila C/EBP. Cell 71, 51-62.

Montell, D.J., Yoon, W.H., and Starz-Gaiano, M. (2012). Group choreography: mechanisms orchestrating the collective movement of border cells. Nat. Rev. Mol. Cell Biol. 13, 631-645.

Morais-de-Sá, E., Mirouse, V., and St Johnston, D. (2010). aPKC phosphorylation of Bazooka defines the apical/lateral border in Drosophila epithelial cells. Cell 141, 509-523.

Murphy, A.M., and Montell, D.J. (1996). Cell typespecific roles for Cdc42, Rac, and RhoL in Drosophila oogenesis. J. Cell Biol. 133, 617-630.

Nance, J., and Zallen, J.A. (2011). Elaborating polarity: PAR proteins and the cytoskeleton. Development 138, 799-809.

Niessen, M.T., Iden, S., and Niessen, C.M. (2012). The in vivo function of mammalian cell and tissue polarity regulators--how to shape and maintain the epidermal barrier. J. Cell Sci. 125, 3501-3510.

Niewiadomska, P., Godt, D., and Tepass, U. (1999). DE-Cadherin is required for intercellular motility during Drosophila oogenesis. J. Cell Biol. 144, 533-547.

Pinheiro, E.M., and Montell, D.J. (2004). Requirement for Par-6 and Bazooka in Drosophila border cell migration. Development 131, 5243-5251.

Ridley, A.J. (2015). Rho GTPase signalling in cell migration. Curr. Opin. Cell Biol. 36, 103-112.

Santoni, M.-J., Kashyap, R., Camoin, L., and Borg, J.P. (2020). The Scribble family in cancer: twentieth anniversary. Oncogene 39, 7019-7033.

Scarpa, E., and Mayor, R. (2016). Collective cell migration in development. J. Cell Biol. 212, 143155.

Stuelten, C., Parent, C.A., and Montell, D.J. (2017). Cell Motility in Cancer Metastasis: Mechanistic 
Insights from Simple Model Organisms. Nature Reviews Cancer.

St Johnston, D., and Ahringer, J. (2010). Cell polarity in eggs and epithelia: parallels and diversity. Cell 141, 757-774.

Szafranski, P., and Goode, S. (2004). A Fasciclin 2 morphogenetic switch organizes epithelial cell cluster polarity and motility. Development 131, 2023-2036.

Tepass, U. (2012). The apical polarity protein network in Drosophila epithelial cells: regulation of polarity, junctions, morphogenesis, cell growth, and survival. Annu. Rev. Cell Dev. Biol. 28, 655-685.

Thiery, J.P., Acloque, H., Huang, R.Y.J., and Nieto, M.A. (2009). Epithelial-mesenchymal transitions in development and disease. Cell 139, 871-890.

Thompson, B.J. (2013). Cell polarity: models and mechanisms from yeast, worms and flies. Development 140, 13-21.

Wang, H., Qiu, Z., Xu, Z., Chen, S.J., Luo, J., Wang, $X$., and Chen, J. (2018). aPKC is a key polarity determinant in coordinating the function of three distinct cell polarities during collective migration. Development 145.

Wang, X., He, L., Wu, Y.I., Hahn, K.M., and Montell, D.J. (2010). Light-mediated activation reveals a key role for Rac in collective guidance of cell movement in vivo. Nat. Cell Biol. 12, 591-597.

Weber, G.F., Bjerke, M.A., and DeSimone, D.W. (2012). A mechanoresponsive cadherin-keratin complex directs polarized protrusive behavior and collective cell migration. Dev. Cell 22, 104-115.

Wodarz, A., and Näthke, I. (2007). Cell polarity in development and cancer. Nat. Cell Biol. 9, 10161024.

Zegers, M.M., and Friedl, P. (2014). Rho GTPases in collective cell migration. Small GTPases 5, e28997.

Zhao, M., Szafranski, P., Hall, C.A., and Goode, S. (2008). Basolateral junctions utilize warts signaling to control epithelial-mesenchymal transition and proliferation crucial for migration and invasion of Drosophila ovarian epithelial cells. Genetics 178, 1947-1971. 